\title{
Building a Holographic Superconductor with Higher-derivative Couplings
}

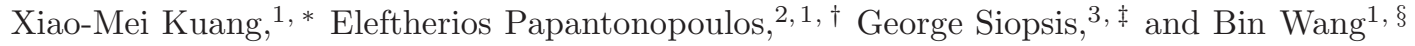 \\ ${ }^{1}$ INPAC, Department of Physics and Shanghai Key Lab for Particle Physics and Cosmology, \\ Shanghai Jiao Tong University, Shanghai 200240, China. \\ ${ }^{2}$ Department of Physics, National Technical University of Athens, GR-15780 Athens, Greece. \\ ${ }^{3}$ Department of Physics and Astronomy, The University of Tennessee, Knoxville, TN 37996 - 1200, USA.
}

(Dated: October 2013)

\begin{abstract}
We discuss the gravitational dual of a holographic superconductor consisting of a $U(1)$ gauge field, a complex scalar field coupled to a charged AdS black hole and a higher-derivative coupling between the $U(1)$ gauge field and the scalar with coupling constant $\eta$. In the presence of a magnetic field, the system possesses localized spatially dependent droplet solutions which, in the low temperature limit, have smaller critical temperature for $\eta>0$ than the droplet solutions without the interaction term $(\eta=0)$. In the weak magnetic field limit, the opposite behavior is observed: the critical temperature increases as we increase $\eta$. We also calculate the energy gap in the probe limit and find that it is larger for $\eta<0$ than the energy gap in the conventional case $(\eta=0)$.
\end{abstract}

PACS numbers: 11.25.Tq, 04.70.Bw, 74.20.-z

\section{INTRODUCTION}

The gauge/gravity duality is a powerful method of studying strongly coupled phenomena using dual weakly coupled gravitational systems [1]. This duality which can be considered as one of the most successful applications of string theory. One of the interesting applications is on condensed matter systems (for a review, see [2]). In trying to understand condensed matter phenomena, e.g., superconductivity, using the gauge/gravity duality, two main approaches have been followed. One is a more phenomenological approach (termed bottomup approach), in which terms in the action are postulated and their effects studied, postponing the derivation of such terms and their relative strength from a full quantum theory to the future. In the other approach (termed top-down approach), one starts with the full quantum theory (string/M theory) and uses it to derive all terms in the action. Although the latter is a more rigorous approach, the range of applications is limited. In our view, progress needs to be made in both directions.

In the bottom-up approach, the simplest holographic superconductor model is described by an EinsteinMaxwell-scalar field theory with a negative cosmological constant [3, 4]. The gravity dual of the holographic superconductor is an Abelian-Higgs model with a stationary black hole metric in which the scalar field condenses below a certain critical temperature. The mechanism of instability has a geometric origin [5, 6]. If the charge of the scalar field is large enough, then the effective mass of the scalar field $m_{\text {eff }}^{2}=m^{2}+q^{2} g^{t t} A_{t}^{2}$, where $A_{t}$ is the electrostatic potential, can drop below the Breitenlohner-Freedman bound near the horizon of the black hole signaling the breaking of an Abelian gauge symmetry outside the black hole horizon. Since the space is asymptotically AdS, the scalar field is trapped outside the black hole horizon resulting in the formation of a condensate which destabilizes the configuration, and a hairy black hole forms below the critical temperature (for a review, see [7], and references therein).

This phenomenological approach has the virtue of simplicity, but does not capture all the underlying features of the gauge/gravity duality including quantum effects. This is hard to implement, as it remains a challenge to embed this model in a quantum system (string/M-theory). In the top-down approach, one aims at finding exact solutions of $D=11$ and type IIB supergravity. The latter approach is mainly based on KaluzaKlein truncations of supergravity theories. Fully back reacted solutions of $D=11$ supergravity describing holographic superconductors in three spacetime dimensions have been found [8, 9]. These models, which are consistent truncations of string/M theories reduced to four spacetime dimensions, contain a large number of

\footnotetext{
*Electronic address: kuangxiaomei@sjtu.edu.cn

$\dagger$ Electronic address: lpapa@central.ntua.gr

‡Electronic address: siopsis@tennessee.edu

$\S$ Electronic address: wang b@sjtu.edu.cn
} 
scalar, gauge fields and high derivatives of them, which need to be constrained in order to make the models tractable [10].

Interesting physics arises when an external magnetic field is applied to a superconductor. As the temperature is lowered and the external magnetic field becomes stronger, the superconductors expel the magnetic field. This is the well-known Meissner effect. Depending on their behavior in the presence of an external magnetic field, ordinary superconductors are classified into two categories, namely type I and type II. In type I superconductors, for fields stronger than a critical value $\left(B>B_{c}\right)$, a first-order transition occurs from the superconducting phase to the normal phase. On the other hand, in type II superconductors, a gradual second-order phase transition occurs, and the material ceases to be a superconductor for $B>B_{c 2}$, where $B_{c 2}$ is the upper critical field strength.

Holographic superconductors in the presence of external magnetic field have been discussed in the bottomup approach [11 14]. It was found that for a non-zero external magnetic field in two spatial dimensions, it is inconsistent to have non-trivial spatially independent solutions on the boundary. Two classes of localized solutions were found, the droplet [15], and vortex solutions with integer winding number [16 18].

In the top-down approach, the effects of an external magnetic field were discussed in [19, 20]. A model was proposed [19] which had an explicit field theory realization as strongly coupled $\mathcal{N}=2$ super Yang-Mills theory with flavor. Using gauge/gravity duality, with the probe of two flavor D7-branes in the AdS black hole background, it was shown that the system underwent a second-order phase transition to a new state which was a p-wave superconductor because of the presence of flavor symmetry. Subsequently in [20], the Meissner effect was studied by introducing an external magnetic field.

In all the above approaches, the effects of an external magnetic field on the holographic superconductor were considered within the standard Maxwell electrodynamics and coupling to charges. There are various reasons to consider higher-derivative terms as corrections to the usual Maxwell field couplings. First of all these couplings appear in a consistent truncation of string/M theories. Secondly, using the usual Maxwell theory it is widely believed that holographic superconductors are of type II rather than type I. It would be interesting to see if these terms affect the behavior of holographic superconductor under the influence of an external magnetic field. In this direction [21] (see also [22]), by introducing higher-derivative corrections to the usual Maxwell action, it was found that the value of the critical field strength $B_{c}$ was affected, indicating that the presence of these terms may alter the nature of the phase transition from the superconducting to the normal state. Further work is needed to understand whether these terms can give a first-order transition.

Our main motivation to consider higher-order terms comes from the recent progress in high- $T_{c}$ superconductors. It was found that, when a strong external magnetic field coupled to the spins of the conduction electrons is applied to a superconductor, inhomogeneous phases appear as the temperature is lowered. This results in a separation of the Fermi surfaces corresponding to electrons with opposite spins (for a review see [23]), and the electron pairing is destroyed, resulting in a first-order transition from the superconducting state to the normal state. As has been shown by Fulde and Ferrell [24], and Larkin and Ovchinnikov [25], a new state (the FFLO state) can form with a modulated order parameter in a weakly coupled BCS system.

A way to understand the formation of these inhomogeneous states in a strongly coupled system is to generalize the potential of holographic superconductors by including terms containing higher-derivative couplings of the scalar field to the gauge potential. Such terms should arise from quantum corrections in a top-down approach. In the bottom-up approach followed here, we are led to consider the effective potential

$$
V(\Psi)=m^{2}\left|\Psi^{2}\right|+\eta\left|F^{\mu \nu} D_{\nu} \Psi\right|^{2}+\eta^{\prime}\left|F_{\mu \nu} D^{\mu}\left(F^{\nu \kappa} D_{\kappa} \Psi\right)\right|^{2}+\ldots,
$$

where $F=d A$ is the field strength of a $\mathrm{U}(1)$ gauge field $A_{\mu}, \Psi$ is a charged complex scalar field of mass $m$ and charge $q$, and $D_{\mu}=\nabla_{\mu}-i q A_{\mu}$. In this paper, we limit ourselves to the case in which all couplings, except $\eta$, are set to zero $\left(\eta^{\prime}=\cdots=0\right)$, leaving a more complete discussion to future work. We study the effects of allowing the gauge field to develop both an electric and a magnetic component. In the absence of the higher-order interaction $(\eta=0)$, we reproduce the droplet solution discussed in [16]. In the case of non-vanishing coupling constant $\eta$, we find that, as the magnetic field becomes stronger, the droplet solution becomes more inhomogeneous. Moreover, as the temperature is lowered, there is a critical value $B_{c}$ of the magnetic field above which the critical temperature is higher than in a system with $\eta=0$.

In the case of weak magnetic field, and with $\eta=0$, we reproduce the results of Ref. [3]. If we switch on the higher-derivative coupling, we find that the system undergoes a phase transition at a higher critical temperature. This gives an interesting and unexpected result: as the strength of the higher-derivative interaction increases, the gap becomes smaller. We find a gap $E_{g} \sim 0.6 T_{c}$ compared to $E_{g} \sim 0.8 T_{c}$ when $\eta=0$. Recently, there has been a renewed interest in this problem. In [26], a periodic potential was introduced, and a value of $E_{g} \sim 0.4 T_{c}$ was found, which is closer to the experimental value. It would be interesting to see if we can attain this value in our model as well, by tuning the coupling constant $\eta$. 
The paper is organized as follows. In section III we present the details of the model. In section III we consider droplet solutions, and calculate the critical temperature with the electromagnetic field having both electric and magnetic components. In section IV we go below the critical temperature assuming that the magnetic field is weak and calculate the energy gap in the probe limit. Finally in section $\nabla$, we discuss our conclusions.

\section{THE MODEL}

We consider the action

$$
S=\int d^{4} x \sqrt{-g}\left[\frac{R+6 / L^{2}}{16 \pi G}-\frac{1}{4} F_{\mu \nu} F^{\mu \nu}-\left|D_{\mu} \Psi\right|^{2}-V(\Psi)\right],
$$

where the potential term is given by (10) with $\eta^{\prime}=\cdots=0$. The field equations are:

- the Einstein equations

$$
R_{\mu \nu}-\frac{1}{2} R g_{\mu \nu}-\frac{3}{L^{2}} g_{\mu \nu}=8 \pi G T_{\mu \nu}
$$

- the Maxwell equations

$$
\begin{aligned}
\nabla_{\mu} F^{\mu \nu} & +\frac{\eta}{\sqrt{-g}} \partial_{\mu}\left[\sqrt{-g}\left(D_{\kappa} \Psi\right)\left(D_{\lambda} \Psi\right)^{*}\left(g^{\kappa \nu} F^{\mu \lambda}-g^{\kappa \mu} F^{\nu \lambda}+g^{\nu \lambda} F^{\mu \kappa}-g^{\mu \lambda} F^{\nu \kappa}\right)\right] \\
& =i q\left[\Psi^{*}\left(D^{\nu} \Psi\right)-\Psi\left(D^{\nu} \Psi\right)^{*}\right]+i q \eta g_{\mu \rho} F^{\rho \nu}\left[F^{\mu \kappa} \Psi^{*}\left(D_{\kappa} \Psi\right)-F^{\mu \lambda} \Psi\left(D_{\lambda} \Psi\right)^{*}\right]
\end{aligned}
$$

- and the scalar field equation

$$
\begin{aligned}
-\frac{1}{\sqrt{-g}} \partial_{\mu} & {\left[\sqrt{-g} g^{\mu \nu}\left(\partial_{\nu} \Psi-i q A_{\nu} \Psi\right)\right]+i q g^{\mu \nu} A_{\nu}\left(\partial_{\mu} \Psi-i q A_{\mu} \Psi\right)+m^{2} \Psi } \\
& -\frac{\eta}{\sqrt{-g}} \partial_{\mu}\left[\sqrt{-g} g_{\kappa \lambda} F^{\kappa \nu} F^{\lambda \mu}\left(\partial_{\nu} \Psi-i q A_{\nu} \Psi\right)\right]+i q \eta g_{\kappa \lambda} F^{\kappa \nu} F^{\lambda \mu} A_{\mu}\left(\partial_{\nu} \Psi-i q A_{\nu} \Psi\right)=0 .
\end{aligned}
$$

In this work, we will set $L=1,8 \pi G=1, q=1$.

We note that the presence of the coupling constant $\eta$ adds new terms in the field equations which makes the system of the differential equations highly non-trivial. However, in spite of the complexity of the system, we were able to perform numerical calculations and also calculate the critical temperature analytically in the weak field limit.

\section{THE CRITICAL TEMPERATURE}

In this section, we switch on a magnetic field and study the effect of spatially dependent solutions on the critical temperature.

Spatially dependent solutions in the presence of a magnetic field were studied in [11, 15, 16]. Solving the Maxwell-scalar equations in a dyonic black hole background localized droplet and vortex solutions were found and their $(B, T)$ phase diagram was studied. In [4], with the addition of an external magnetic field, it was argued that the holographic superconductor is of type II as the magnetic field is lowered. An analytic study of a holographic superconductor was carried out in [12], where the upper critical magnetic field was calculated analytically. Vortex lattice solutions in the presence of a magnetic field were presented in [18].

To study the effect of the interaction term (with $\eta \neq 0$ ), we will consider the backreaction of the Maxwell field to gravity. The Einstein-Maxwell field equations (3) and (4) at the critical temperature are solved by a dyonic black hole (i.e., one with both an electric and a magnetic charge). The metric reads [27]

$$
d s^{2}=\frac{d r^{2}}{r^{2} h(r)}+r^{2}\left[-h(r) d t^{2}+d x^{2}+d y^{2}\right] \quad, \quad h(r)=1-\frac{r_{+}^{3}}{r^{3}}+\frac{\left(\lambda^{2}+\mathcal{B}^{2}\right) r_{+}^{4}}{4 r^{3}}\left(\frac{1}{r}-\frac{1}{r_{+}}\right) .
$$

The gauge field is given by

$$
A_{\mu}=A_{t}(r) d t+A_{y}(x) d y=\lambda r_{+}\left(1-\frac{r_{+}}{r}\right) d t+\mathcal{B} r_{+}^{2} x d y,
$$


where according to the AdS/CFT dictionary, the charge density and the chemical potential on the boundary are given by $\rho=\lambda r_{+}^{2}$ and $\mu=\lambda r_{+}$, respectively. $\mathcal{B} r_{+}^{2}$ is the magnitude of the external magnetic field (in the radial direction, $r$, perpendicular to the boundary $x y$-plane). At the horizon, $r=r_{+}$, we have $A_{t}=0$. The Hawking temperature of the black hole is

$$
T=\frac{3 r_{+}}{4 \pi}\left(1-\frac{\lambda^{2}+\mathcal{B}^{2}}{12}\right) .
$$

In the above background with a magnetic field $\mathcal{B}$, we will investigate the spatially dependent solutions of the field equations by considering the ansatz $\Psi=\Psi(r, x)$. Near the AdS boundary, the asymptotic behavior is $\Psi=\frac{\Psi_{1}}{r^{\Delta_{-}}}+\frac{\Psi_{2}}{r^{\Delta_{+}}}$with $\Delta_{ \pm}=\frac{3}{2} \pm \frac{1}{2} \sqrt{9+4 m^{2}}$. Both $\Psi_{1}$ and $\Psi_{2}$ correspond to normalizable modes, therefore we obtain two different systems (labeled by $\Delta_{ \pm}$) in which one mode is the source and the other gives the vacuum expectation value of the dual operator of dimension $\Delta_{i}\left(\Psi_{i} \sim\left\langle\mathcal{O}_{i}\right\rangle\right)$.

Right below the critical temperature, we may regard the scalar field as a perturbation in the gravitational background of (6) and (17). Then the scalar field equation in the coordinate $z=\frac{r_{+}}{r}$ becomes

$$
\begin{aligned}
& {\left[1-\frac{\eta}{r_{+}^{2}} z^{4} A_{t}^{\prime 2}\right] \Psi^{\prime \prime}+\left[\left(\frac{h^{\prime}}{h}-\frac{2}{z}\right)-\frac{\eta}{r_{+}^{2}} z^{4}\left(\frac{h^{\prime}}{h}+\frac{2}{z}\right) A_{t}^{\prime 2}-\frac{2 \eta}{r_{+}^{2}} z^{4} A_{t}^{\prime} A_{t}^{\prime \prime}\right] \Psi^{\prime}} \\
& +\frac{1+\eta \mathcal{B}^{2} z^{4}}{h} \partial_{x}^{2} \Psi+\left[-\frac{m^{2}}{z^{2}}+\frac{1-\frac{\eta}{r_{+}^{2}} z^{4} A_{t}^{\prime 2}}{r_{+}^{2} h} A_{t}^{2}-\mathcal{B}^{2} x^{2}-\eta \mathcal{B}^{4} z^{4} x^{2}\right] \frac{1}{h} \Psi=0,
\end{aligned}
$$

where the prime denotes differentiation with respect to $z$. Without loss of generality, we can take $\Psi$ to be real and separate $\Psi$ into functions of a single variable,

$$
\Psi(z, x)=\Psi_{1}(z) \Psi_{2}(x)
$$

Combining equations (9) and (10), we obtain

$$
\begin{aligned}
& h\left[1-\frac{\eta}{r_{+}^{2}} z^{4} A_{t}^{\prime 2}\right] \Psi_{1}^{\prime \prime}+\left[\left(\frac{h^{\prime}}{h}-\frac{2}{z}\right)-\frac{\eta}{r_{+}^{2}} z^{4}\left(\frac{h^{\prime}}{h}+\frac{2}{z}\right) A_{t}^{\prime 2}-\frac{2 \eta}{r_{+}^{2}} z^{4} A_{t}^{\prime} A_{t}^{\prime \prime}\right] h \Psi_{1}^{\prime} \\
& \quad+\left[-\frac{m^{2}}{z^{2}}+\frac{1-\frac{\eta}{r_{+}^{2}} z^{4} A_{t}^{\prime 2}}{r_{+}^{2} h} A_{t}^{2}\right] \Psi_{1}+\frac{\Psi_{1}\left(1+\eta \mathcal{B}^{2} z^{4}\right)}{\Psi_{2}}\left[\Psi_{2}^{\prime \prime}-\mathcal{B}^{2} x^{2} \Psi_{2}\right]=0 .
\end{aligned}
$$

For consistency of this equation, we need

$$
\Psi_{2}^{\prime \prime}-\mathcal{B}^{2} x^{2} \Psi_{2}=-k^{2} \Psi_{2}
$$

where $k^{2}$ is an arbitrary parameter. This equation is of the form of Schrödinger's equation for a simple harmonic oscillator. The eigenvalues are

$$
k^{2}=(2 n+1) \mathcal{B}, \quad n=0,1,2, \ldots,
$$

and the corresponding eigenfunctions are given in terms of Hermite polynomials, $H_{n}(\sqrt{2 \mathcal{B}} x)$. Note that the $x$-dependent part of the scalar field obeys the same equation as the one found in [15]. As explained in [15], we ought to choose the ground state $(n=0)$ to find the critical temperature.

Substituting the $x$-dependent equation (12) into (11), we obtain the field equation for $\Psi_{1}(z)$ which reads

$$
\begin{aligned}
& {\left[1-\eta \lambda^{2} z^{4}\right] \Psi_{1}^{\prime \prime}+\left[\left(\frac{h^{\prime}}{h}-\frac{2}{z}\right)-\eta \lambda^{2} z^{4}\left(\frac{h^{\prime}}{h}+\frac{2}{z}\right)\right] \Psi_{1}^{\prime}} \\
& +\left[\frac{2}{z^{2} h}+\frac{\lambda^{2}\left(1-\eta \lambda^{2} z^{4}\right)(1-z)^{2}}{h^{2}}-\frac{\mathcal{B}\left(1+\eta \mathcal{B}^{2} z^{4}\right)}{h}\right] \Psi_{1}=0
\end{aligned}
$$

where we have chosen $m^{2}=-2$ for definiteness, and expressed $A_{t}$ using (7).

Observe that new $\eta$-dependent terms appear in the field equation for the scalar field compared to the scalar equation in [15]. They give a different $z$-dependent behavior of the scalar field, as we will discuss below.

The asymptotic boundary condition $(z \rightarrow 0)$ becomes

$$
\Psi_{1}(z)=\psi_{1} z+\psi_{2} z^{2}
$$



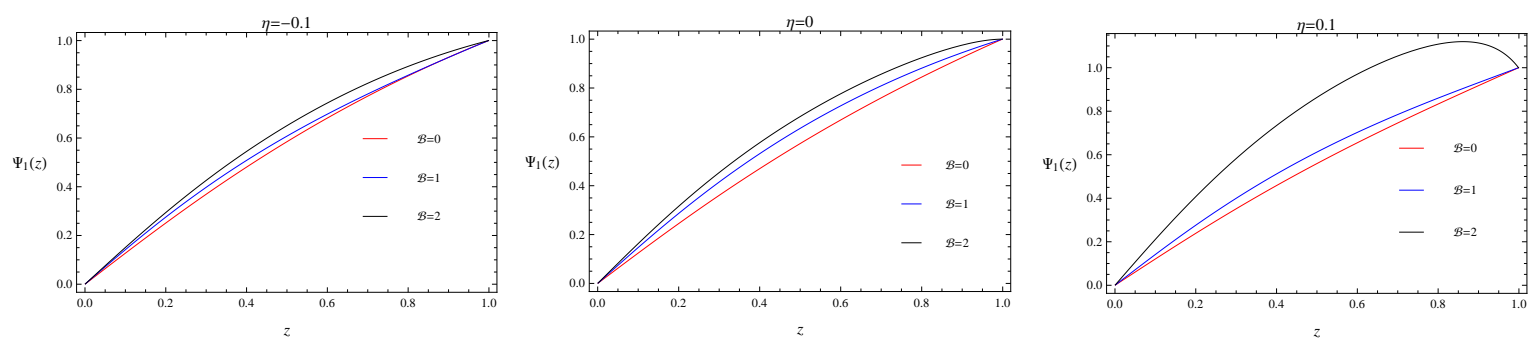

FIG. 1: The $z$-dependence of the scalar field for various values of the magnetic field $\mathcal{B}$ and coupling constant $\eta$.

At the horizon, we have the regularity condition

$$
\Psi_{1}^{\prime}(1)=\frac{2-\mathcal{B}\left(1+\eta \mathcal{B}^{2}\right)}{\left(3-\frac{\lambda^{2}+\mathcal{B}^{2}}{4}\right)\left(1-\eta \lambda^{2}\right)} \Psi_{1}(1)
$$

We will investigate the $z$-dependent profile of the scalar field perturbation, solving equation (14) focusing on the case of scaling dimension $\Delta=1$ (setting $\psi_{2}=0$ ). For a numerical solution, we impose the boundary condition $\Psi_{1}(1)=1$, and solve (14) with the use of a shooting method. Notice that the boundary condition at the horizon (16) depends on the coupling constant $\eta$. The $z$-dependence of the scalar field for different values of the magnetic field is shown in Fig. 1. We see that as the magnetic field becomes stronger, the corresponding scalar perturbation in the bulk becomes bigger. This behavior is enhanced by the increasing strength of the interaction.

In Fig. 2 we plot the phase diagram of the critical magnetic field and critical temperature. Above the lines, the holographic system will change into the normal phase. We use the critical temperature $T_{0}$ for $\mathcal{B}=0, \eta=0$ as a scale in the figure. The phase diagram reveals some very interesting features.

Stronger magnetic fields correspond to lower critical temperatures. This behavior is expected, and for $\eta=0$ it has been discussed in [18, 28]. However in our case, as the strength of the higher-derivative coupling increases, smaller critical temperatures at strong enough magnetic field can be probed as can be seen on the right panel of Fig. 2. To see the behavior of the system at the quantum critical point $(T=0)$, we need to solve the full back-reacted system of Einstein-Maxwell-scalar field equations. Note that as the coupling $\eta$ gets larger, for fixed critical temperature, the critical magnetic field $B_{c}$, above which the superconductor enters its normal phase, decreases.

So far we have concentrated on the strong magnetic field regime exhibited in Fig. 22 Let us now look at the left panel of Fig. 2 in the low magnetic field regime. We observe that, unlike strong magnetic fields, the critical temperature is larger if the higher-derivative coupling is stronger. This is consistent with the results obtained below in section IV There is a transition region marked in the inserted box in the left part of Fig. 2. which we enlarge in Fig. 3 . We find that the cross point between lines of $\eta=-0.1$ and $\eta=0$ occurs at $\mathcal{B} \approx 0.8124$. Below this value of the magnetic field, the critical temperature is smaller for negative coupling $\eta$. Above this value, the critical temperature for $\eta=-0.1$ becomes higher than that of the minimal coupling. The cross point between lines of $\eta=-0.1$ and $\eta=0.1$ appears at $\mathcal{B} \approx 0.8268$. Above this transitional strength of the magnetic field, the critical temperature for $\eta=-0.1$ is higher than the positive coupling. The transitional strength of the magnetic field between $\eta=0.1$ and the minimal coupling is at $\mathcal{B} \approx 0.8407$, above which the critical temperature for minimal coupling becomes higher.

One of the reasons of introducing the generalized potential term was to account for the FFLO states. These states appear in high-field superconductors when a strong magnetic field is applied, as Fulde and Ferrell [24] and Larkin and Ovchinnikov [25] showed independently. Their main feature is exhibiting an order parameter which is not a constant, but has a space variation. Unfortunately, we cannot compare the transition temperature of our inhomogeneous solution to that of a homogeneous solution, because the latter does not exist in our system. ${ }^{1}$ We will comment further on this point in section $\mathrm{V}$

${ }^{1}$ It is possible to compare homogeneous and inhomogeneous solutions in the presence of a magnetic field, if we introduce two gauge fields, as was discussed in [29, 30]. The first gauge field couples to the scalar sourcing a charge condensate below a critical critical temperature, whereas the second gauge field incorporates a magnetic field that couples to the spin in the boundary theory. 

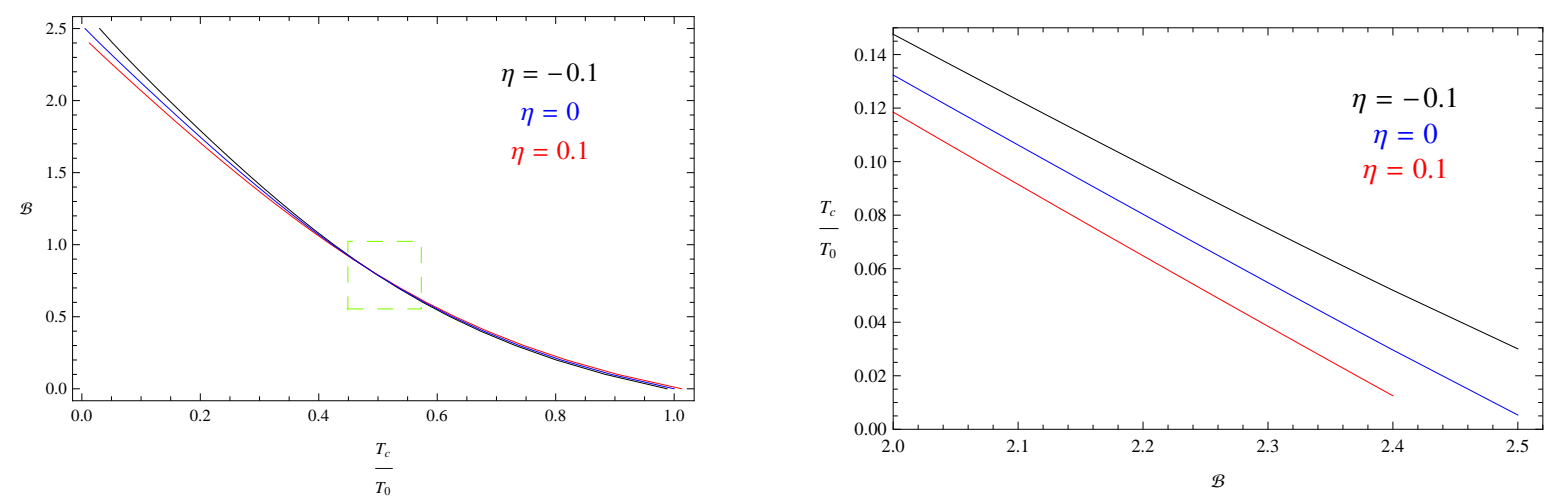

FIG. 2: The critical temperature as a function of magnetic field. The low $\mathrm{T}$ behavior is enlarged in the right panel. The enlarged view in the green rectangle region is shown in Fig. 3

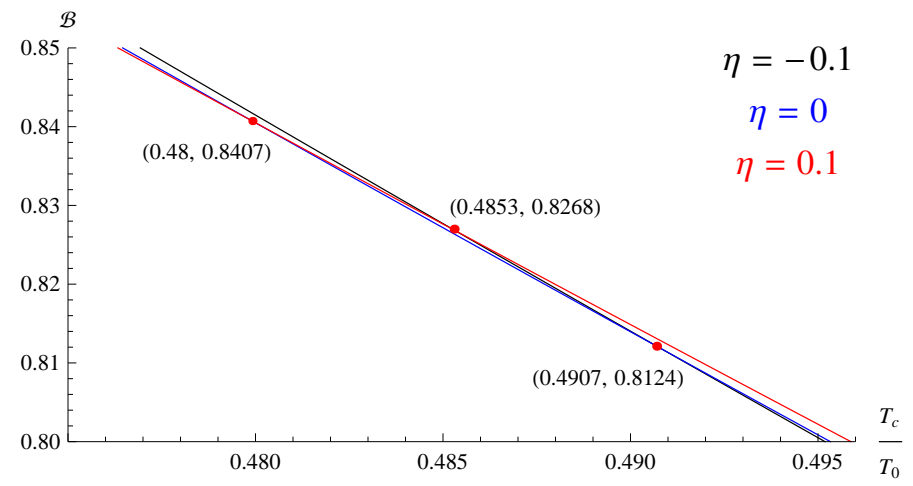

FIG. 3: The critical temperature as a function of magnetic field in the green rectangle region of Fig. 2

In the above calculations, we have considered an exact solution of the field equations (dyonic black hole), which includes the back-reaction of the electromagnetic field. Thus, our results for the critical temperature are exact in the entire parameter space depicted in Fig. 2, As we lower the temperature below the critical temperature, the field equations become considerably more involved. We shall concentrate on the case of a weak magnetic field where the backreaction on the metric can be safely ignored. In this regime, the critical temperature is finite and there is a region below $T_{c}$ which is well above zero temperature so that the nobackreaction approximation remains valid. In the strong magnetic field limit, there is no region below $T_{c}$ where the backreaction can be ignored, because the critical temperature is already close to zero.

\section{BELOW THE CRITICAL TEMPERATURE}

In this section we perform calculations below the critical temperature. We concentrate on the weak magnetic field limit. To simplify the calculations, we also focus on the probe limit by ignoring the back-reaction to the metric. Thus the metric takes the form of a planar Schwarzschild AdS black hole,

$$
d s^{2}=\frac{1}{z^{2}}\left[-h(z) d t^{2}+\frac{d z^{2}}{h(z)}+d x^{2}+d y^{2}\right] \quad, \quad h(z)=1-z^{3} .
$$

We will study the condensation of the scalar field and calculate the critical temperature both analytically and numerically in the weak magnetic field limit $A_{i} \rightarrow 0(i=1,2,3)$. This results in a solution which is approximately spatially independent. Thus, we consider a spherically symmetric ansatz

$$
\Psi=\Psi(z), \quad A_{\mu}=A_{t}(z) d t
$$

Note that the potential term (11) in the weak magnetic field limit $(\mathcal{B} \rightarrow 0)$ only contains gradients in the radial direction. Therefore, it merely induces interactions between the electric field and the scalar field. 

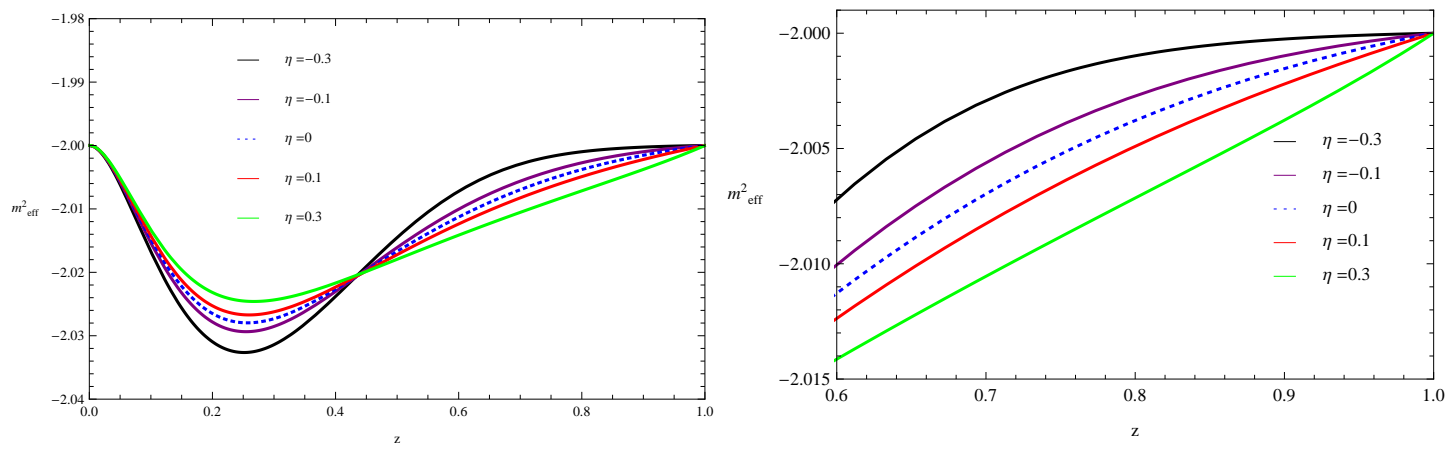

FIG. 4: The value of the effective mass of the scalar field 21) as a function of $z$ for scaling dimension $\Delta=1$ and various values of the coupling $\eta$.

The Maxwell equation (4) and the scalar field equation (5) in the black hole background (17) reduce to, respectively,

$$
\begin{array}{r}
A_{t}^{\prime \prime}+\left[\frac{\frac{2 \eta}{r_{+}^{2}} z^{3} \Psi A_{t}^{2}\left(z \Psi h^{\prime}-2 h\left(\Psi+z \Psi^{\prime}\right)\right)}{-\frac{2 \eta}{r_{+}^{2}} z^{4} h \Psi^{2} A_{t}^{2}+z^{2} h^{2}\left(1+2 \eta z^{2} h \Psi^{\prime 2}\right)}+\frac{2 \eta z^{3} h \Psi^{\prime}\left(z h^{\prime} \Psi^{\prime}+2 h\left(\Psi^{\prime}+z \Psi^{\prime \prime}\right)\right)}{-\frac{2 \eta}{r_{+}^{2}} z^{4} \Psi^{2} A_{t}^{2}+z^{2} h\left(1+2 \eta z^{2} h \Psi^{\prime 2}\right)}\right] A_{t}^{\prime} \\
-\left[\frac{2 \Psi^{2}\left(1+\frac{\eta}{r_{+}^{2}} z^{4} A_{t}^{\prime 2}\right)}{-\frac{2 \eta}{r_{+}^{2}} z^{4} \Psi^{2} A_{t}^{2}+z^{2} h\left(1+2 \eta z^{2} h \Psi^{\prime 2}\right)}\right] A_{t}=0, \\
\Psi^{\prime \prime}+\left[\frac{h^{\prime}}{h}-\frac{2\left(1+\frac{\eta}{r_{+}^{2}} z^{4} A_{t}^{\prime 2}+\frac{\eta}{r_{+}^{2}} z^{5} A_{t}^{\prime} A_{t}^{\prime \prime}\right)}{z\left(1-\frac{\eta}{r_{+}^{2}} z^{4} A_{t}^{\prime 2}\right)}\right] \Psi^{\prime}+\left[\frac{A_{t}^{2}}{r_{+}^{2} h^{2}}-\frac{m^{2}}{z^{2} h\left(1-\frac{\eta}{r_{+}^{2}} z^{4} A_{t}^{\prime 2}\right)}\right] \Psi=0,
\end{array}
$$

where the prime denotes differentiation with respect to $z$. Note that for vanishing coupling $\eta$, the above field equations coincide with the corresponding equations in [3].

As in [3], the crucial feature is the last term in (20) which is a direct coupling of the scalar potential to the scalar field. This term comes from the effective mass term which reads ${ }^{2}$

$$
m_{\mathrm{eff}}^{2}=m^{2}-\frac{z^{2} A_{t}^{2}\left(1-\frac{\eta}{r_{+}^{2}} z^{4} A_{t}^{\prime 2}\right)}{r_{+}^{2} h}
$$

and besides the scalar potential it also depends on the coupling constant $\eta$. This is the gravitational analog [ 6 ] of an Abelian $U(1)$ symmetry breaking outside the horizon of the black hole background (17) as the effective mass (21) drops below the Breitenlohner-Freedman bound $m_{\mathrm{BF}}^{2}=-\frac{9}{4}$.

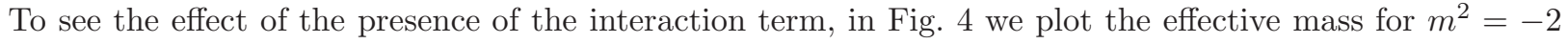
and scaling dimension $\Delta=1$. The left panel shows that large negative values of the coupling constant $\eta$ make $m_{\text {eff }}^{2}$ develop a deeper well. However, as we approach the horizon (right panel) larger values of the coupling $\eta$ give smaller values of the effective mass, which means that the condensation is easier to form. Therefore, the effect of the presence of the interaction term is that as the strength of the coupling $\eta$ is increased, the system undergoes a phase transition in a higher critical temperature as can be seen in Table [. The same behavior can be observed in the case of scaling dimension $\Delta=2$ as can be seen in Fig. 5 and Table II.

Next we will calculate the critical temperature analytically and show that it agrees with our numerical results.

$2 m_{\text {eff }}^{2}$ is the coefficient of $\Psi^{2}$ in the action (2). 

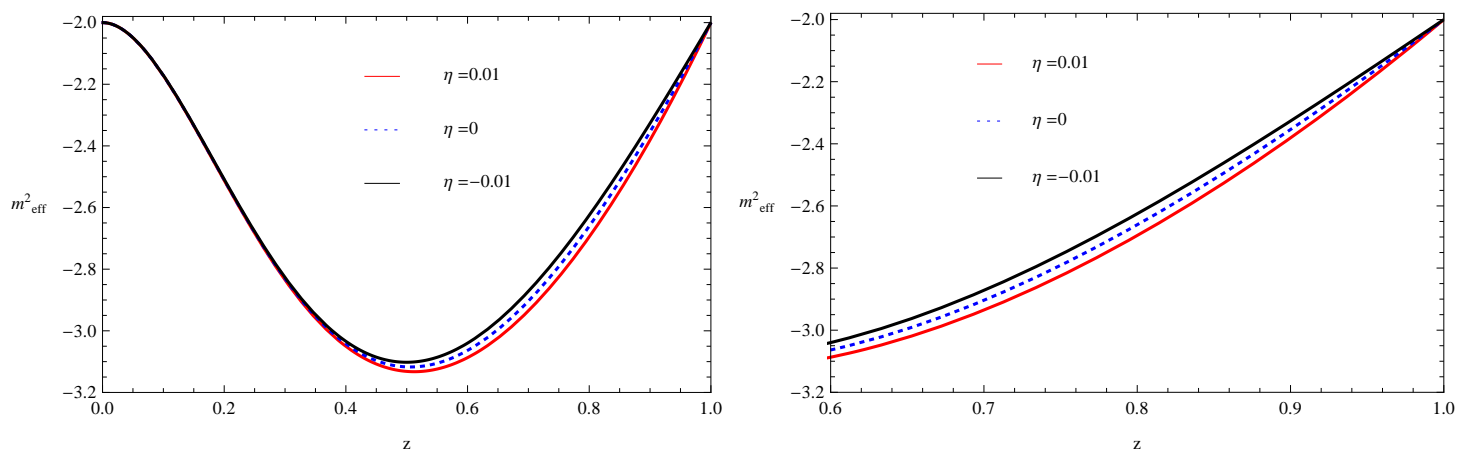

FIG. 5: The value of the effective mass of the scalar field 21) as a function of $z$ for scaling dimension $\Delta=2$ and various values of the coupling $\eta$.

\section{A. Analytic Results}

We will analytically determine the critical temperature for scaling dimension $\Delta=1$ with the use of the method developed in [31]. Since the scalar field $\Psi$ vanishes at the critical temperature $T_{c}$, the Maxwell equation (19) reduces to $A_{t}^{\prime \prime}=0$, which is solved by

$$
A_{t}=\lambda r_{+}(1-z)
$$

where $\lambda r_{+}^{2}$ is the charge density. On the other hand, near the boundary, we can introduce a function $F(z)$ by defining $^{3}$

$$
\Psi=\frac{\left\langle\mathcal{O}_{1}\right\rangle}{\sqrt{2} r_{+}} z F(z)
$$

where $F(z)$ has been normalized as $F(0)=1$ and $F^{\prime}(0)=0$.

Thus, substituting (22) and (23) into the scalar field equation (20) we obtain

$$
\begin{aligned}
F^{\prime \prime}(z) & -\left[\frac{5 z^{3}-2}{z\left(1-z^{3}\right)}+\frac{2\left(1+\eta \lambda^{2} z^{4}\right)}{z\left(1-\eta \lambda^{2} z^{4}\right)}\right] F^{\prime}(z)-\left[\frac{3 z}{1-z^{3}}+\frac{2\left(1+\eta \lambda^{2} z^{4}\right)}{z^{2}\left(1-\eta \lambda^{2} z^{4}\right)}-\frac{2}{z^{2}\left(1-z^{3}\right)\left(1-\eta z^{4} \lambda^{2}\right)}\right] F(z) \\
& +\frac{\lambda^{2}}{\left(1+z+z^{3}\right)^{2}} F=0 .
\end{aligned}
$$

The above equation can be converted into

$$
\left[T(z) F^{\prime}(z)\right]^{\prime}-Q(z) F(z)=0
$$

with

$$
T(z)=\left(1-z^{3}\right)\left(1-\eta \lambda^{2} z^{4}\right), \quad Q(z)=z-\lambda^{2} \frac{(1-z)\left(1-\eta \lambda^{2} z^{4}\right)}{1+z+z^{2}}-\eta \lambda^{2} z^{2}\left(5 z^{3}-2\right) .
$$

With the use of Sturm-Liouville theory, the eigenvalue $\lambda$ can be found as the minimum solution of

$$
\int_{0}^{1} d z\left[T(z) F^{\prime}(z)^{2}+Q(z) F(z)^{2}\right]=0 .
$$

To proceed, we assume the trial function

$$
F(z) \equiv 1-\alpha z^{2}
$$

\footnotetext{
${ }^{3}$ For the other scaling dimension, $\Delta=2$, the definition becomes $\Psi=\frac{\left\langle\mathcal{O}_{2}\right\rangle}{\sqrt{2} r_{+}} z^{2} F(z)$.
} 


\begin{tabular}{|c||c||c||c||c||c||c||c||c|}
\hline$\eta$ & -0.3 & -0.2 & -0.1 & 0 & 0.01 & 0.1 & 0.2 & 0.3 \\
\hline$T_{c} / \sqrt{\rho}$ (numerical) & 0.2224 & 0.2234 & 0.2244 & 0.2255 & 0.2257 & 0.2267 & 0.2280 & 0.2294 \\
\hline$T_{c} / \sqrt{\rho}$ (analytic) & 0.2218 & 0.2228 & 0.2239 & 0.2250 & 0.2251 & 0.2261 & 0.2273 & 0.2287 \\
$(\alpha)$ & $(0.2868)$ & $(0.2720)$ & $(0.2561)$ & $(0.2389)$ & $(0.2371)$ & $(0.2204)$ & $(0.2006)$ & $(0.1793)$ \\
\hline
\end{tabular}

TABLE I: The critical temperature for scaling dimension $\Delta=1$ and various values of the coupling $\eta$.

which satisfies the condition $F(0)=1$ as well as $F^{\prime}(0)=0$. After integrating over $z$ in (27), we find that $\lambda^{2}$ satisfies the second-order equation

$$
a_{2} \lambda^{4}+a_{1} \lambda^{2}+a_{0}=0
$$

where

$$
\begin{aligned}
a_{2}= & \eta\left\{\alpha^{2}\left[2 \sqrt{3} \pi+3\left(-\frac{409}{70}+2 \ln 3\right)\right]+2 \alpha\left[2 \sqrt{3} \pi-3\left(\frac{7}{5}+2 \ln 3\right)\right]+13-12 \ln 3\right\} \\
a_{1}= & \eta \alpha^{2}\left[-\frac{110501}{2310}+6 \sqrt{3} \pi+18 \ln 3\right]+\alpha^{2}(12 \ln 3-13)+\alpha(4 \sqrt{3} \pi+12 \ln 3-36) \\
& +\eta \alpha\left[-\frac{3263}{105}+12 \sqrt{3} \pi-36 \ln 3\right]+3 \eta\left(\frac{97}{7}-12 \ln 3\right)+2 \sqrt{3} \pi-6 \ln 3 \\
a_{0}= & -10 \alpha^{2}+6 \alpha-6 .
\end{aligned}
$$

For a fixed value of the coupling $\eta$, we obtain the minimum value of $\lambda^{2}$ by varying $\alpha$. Then the critical temperature is found to be

$$
T_{c}=\frac{3}{4 \pi} r_{+}=\frac{3}{4 \pi \sqrt{\lambda_{\min }}} \sqrt{\rho} .
$$

With the use of (31), the analytic critical temperatures are summarized in Table प In Table II we also summarize the critical temperature for the case of scaling dimension $\Delta=2$ calculated analytically. We see that the critical temperature increases as the coupling constant $\eta$ increases. This is reasonable, because from the effective mass (21) and Fig. 4 (or 5), we learn that larger $\eta$ corresponds to more negative effective mass, which in turn implies that the system becomes more unstable and the symmetry is easier to be broken.

\section{B. Numerical Results}

The field equations (19) and (20) were solved numerically with $m^{2}=-2$. In Table \ we list the critical temperature calculated numerically for various values of the coupling constant $\eta$, and compare it with the analytic results calculated from Eq. (31). Note that for $\eta=0$ we recover the results of [3]. We also list the corresponding values of $\alpha$. From this table, we can see that the numerical results and the analytic results are in good agreement.

Next we examine the effect of the presence of the higher-derivative coupling term on the condensation of the scalar field. In Fig. [6 we show the vacuum expectation values of the two operators $\mathcal{O}_{1}$ and $\mathcal{O}_{2}$ versus the critical temperature. We see that with the increase of the strength $\eta$ of the interaction, the gap becomes smaller, which means that the scalar operator can condense easier when the coupling $\eta$ is stronger. This agrees with the property of the critical temperature we discussed above. For higher $\eta$, our numerical calculation becomes harder at lower temperature, however this does not prevent us from observing the qualitative influence of the interaction on the condensation. For scaling dimension $\Delta=1$, this behavior is more pronounced (see left panel of Fig. 6). For scaling dimension $\Delta=2$, we enlarge a local region inserted into the right panel of Fig. 6 to bring out this behavior. From the critical temperature behavior obtained with both numerical and analytic methods, together with the observed gap behavior, we can conclude that in the weak magnetic field limit, the greater the strength of the interaction the easier it is for the condensation to form. This implies that in the boundary field theory, which is dual to the gravitational theory, with stronger higher-derivative coupling between the $U(1)$ gauge field and the scalar field, the gauge symmetry can be broken more easily. 

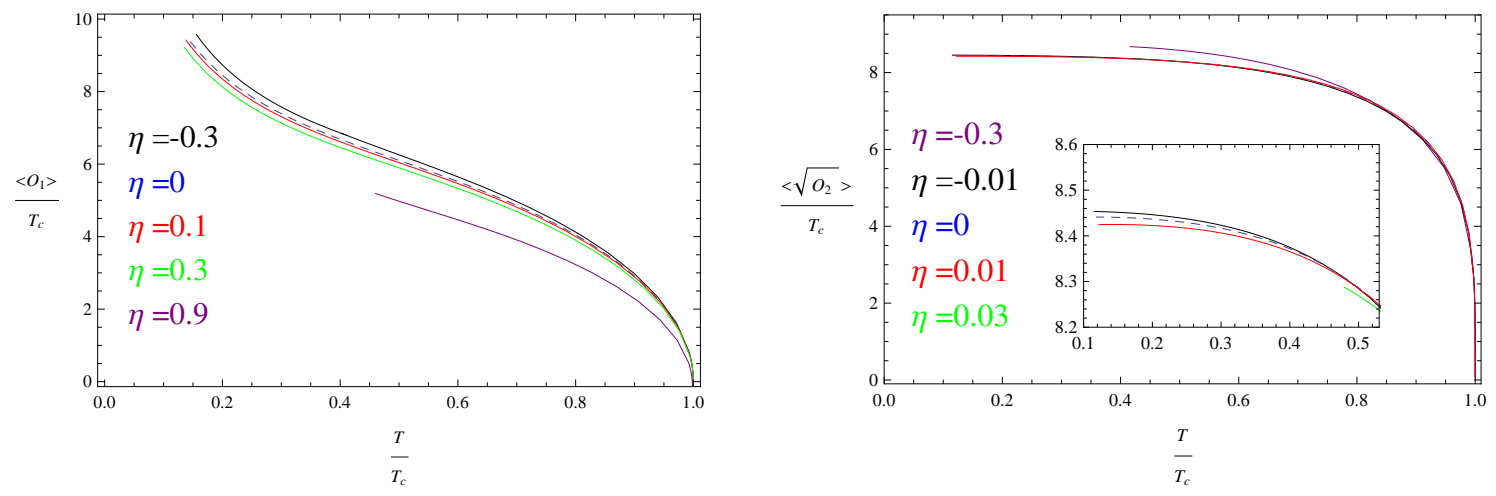

FIG. 6: The order parameters $\left\langle\mathcal{O}_{1}\right\rangle$ (left panel) and $\left\langle\mathcal{O}_{2}\right\rangle$ (right panel) as functions of temperature for various values of the coupling $\eta$.

\begin{tabular}{|c||c||c||c||c||c||c||c||}
\hline$\eta$ & -0.2 & -0.1 & 0 & 0.01 & 0.1 & 0.2 & 0.3 \\
\hline$T_{c} / \sqrt{\rho}$ (analytic) & 0.1092 & 0.1157 & 0.1170 & 0.1170 & 0.1191 & 0.1384 & 0.1506 \\
\hline
\end{tabular}

TABLE II: The critical temperature for scaling dimension $\Delta=2$ and various values of the coupling $\eta$.

\section{DISCUSSION}

We set up a gravitational dual of a holographic superconductor which included a higher-derivative coupling of strength $\eta$. Our system had standard composition, consisting of a $U(1)$ gauge field, and a complex scalar field coupled to gravity. The novel feature was a higher-derivative coupling between the $U(1)$ gauge field and the scalar field. We solved the field equations and compared the results with the conventional case of a holographic superconductor $(\eta=0)$.

In the limit in which the magnetic field is weak, we found a spatially independent (homogeneous) solution. In the case in which the coupling strength $\eta$ of the higher-derivative coupling vanishes, we recovered the results of [3]. As the strength of the coupling increases, the gravitational mechanism of breaking an Abelian $U(1)$ symmetry outside the horizon of a black hole [6] becomes more effective, as can be seen in Fig. 4 and Fig. 5 . Calculating the critical temperature, both analytically and numerically, we found that as the strength of the interaction increased, there was an enhancement of the critical temperature (Tables 【and II) at which the scalar condensate formed.

In the presence of a magnetic field, the normal state corresponds to the background of a dyonic black hole. The field equations possess spatially dependent (inhomogeneous) solutions. We found that the presence of the higher-derivative coupling did not affect the $x$-dependent profile of the scalar field solution. The evolution of the $x$-dependent profile of the scalar field is given by the solution of equation (13) which depends on the strength of the magnetic field. As it is discussed in [15], when the magnetic field is zero, the $x$-dependence of the condensate disappears and we recover the homogeneous solution. However, as the magnetic field is increased, the $x$-dependent profile of the scalar field acquires a "thickness" making the condensate more inhomogeneous.

The presence of the higher-derivative coupling term influences the radial dependence of the scalar field contributing to the critical temperature. In Fig. 2, the $(B, T)$ phase diagram for various values of the coupling $\eta$ is shown. We can see some interesting features of our droplet solutions in the low temperature, strong magnetic field region of the phase diagram (right panel of Fig. 2). For $\eta>0$ and a fixed value of the magnetic field we can probe lower temperatures. For $\eta<0$ the condensate forms at a higher critical temperature and at a stronger magnetic field making the corresponding droplet solution more inhomogeneous. As we discussed, this behavior is reminiscent of the formation of FFLO states, in the case where inhomogeneous states at low temperature are energetically more favorable.

To have a better understanding of our droplet solutions in the $T \rightarrow 0$ limit and their possible connection with the inhomogeneous FFLO states, we have to improve our numerical techniques in order to probe larger (positive and negative) values of the coupling constant $\eta$. We could also look for solutions with a strong magnetic field penetrating the whole $x y$-plane and study the behavior of the system by solving the full non-linear system of the coupled Einstein-Maxwell-scalar equations. Besides the higher-derivative coupling considered in this work, it is also of interest to generalize the study on the influence of other higher-derivative 
terms in the holographic superconductor, especially in the presence of the external magnetic field.

Another interesting problem is to extend this analysis to other types of holographic superconductors which have more structure, e.g., the p-wave superconductor. The motivation is two-fold. Firstly, it would be interesting to see if the effect we found in the s-wave superconductor, namely that the value of the gap is reduced as the strength of the derivative coupling increases, persists in the case of the richer structure of a p-wave superconductor. Secondly, one can study how the presence of the higher derivative terms affects the behavior of a p-wave superconductor as the strength of the magnetic field increases and the temperature is lowered. We have to note however, that even in the s-wave superconductor, the inclusion of the higherderivative term brings technical difficulties so one should expect an increase in the complexity in the case of p-wave superconductor.

\section{Acknowledgments}

This work is supported partially by the NNSF of China and the Shanghai Science and Technology Com-

mission under Grant No. 11DZ2260700. G. S. is supported in part by the US Department of Energy under Grant No. DE-FG05-91ER40627.

[1] J. M. Maldacena, "The large N limit of superconformal field theories and supergravity," Adv. Theor. Math. Phys. 2 (1998) 231 [Int. J. Theor. Phys. 38 (1999) 1113] arXiv:hep-th/9711200.

[2] S. A. Hartnoll, "Lectures on holographic methods for condensed matter physics," Class. Quant. Grav. 26, 224002 (2009) arXiv:0903.3246 [hep-th]].

[3] S. A. Hartnoll, C. P. Herzog and G. T. Horowitz, "Building a Holographic Superconductor," Phys. Rev. Lett. 101, 031601 (2008) arXiv:0803.3295.

[4] S. A. Hartnoll, C. P. Herzog and G. T. Horowitz, "Holographic Superconductors," JHEP 0812, 015 (2008) arXiv:0810.1563.

[5] S. S. Gubser, "Phase transitions near black hole horizons," Class. Quant. Grav. 22, 5121 (2005) hep-th/0505189.

[6] S. S. Gubser, "Breaking an Abelian gauge symmetry near a black hole horizon," Phys. Rev. D 78, 065034 (2008) arXiv:0801.2977 [hep-th]].

[7] G. T. Horowitz, "Theory of Superconductivity," Lect. Notes Phys. 828, 313 (2011) arXiv:1002.1722 [hep-th]].

[8] J. P. Gauntlett, S. Kim, O. Varela and D. Waldram, "Consistent supersymmetric Kaluza-Klein truncations with massive modes," JHEP 0904 (2009) 102 arXiv:0901.0676 [hep-th]].

[9] J. P. Gauntlett, J. Sonner and T. Wiseman, "Holographic superconductivity in M-Theory," Phys. Rev. Lett. 103 (2009) 151601 arXiv:0907.3796 [hep-th]].

[10] J. P. Gauntlett, J. Sonner and T. Wiseman, JHEP 1002, 060 (2010) arXiv:0912.0512 [hep-th]].

[11] T. Albash and C. V. Johnson, "Phases of Holographic Superconductors in an External Magnetic Field," arXiv:0906.0519.

[12] X. -H. Ge, B. Wang, S. -F. Wu and G. -H. Yang, "Analytical study on holographic superconductors in external magnetic field," JHEP 1008, 108 (2010) arXiv:1002.4901.

[13] O. Domenech, M. Montull, A. Pomarol, A. Salvio and P. J. Silva, "Emergent Gauge Fields in Holographic Superconductors," JHEP 1008, 033 (2010) arXiv:1005.1776 [hep-th]].

[14] M. Montull, O. Pujolas, A. Salvio and P. J. Silva, "Magnetic Response in the Holographic Insulator/Superconductor Transition," JHEP 1204, 135 (2012) arXiv:1202.0006 [hep-th]].

[15] T. Albash and C. V. Johnson, "A Holographic Superconductor in an External Magnetic Field," JHEP 0809, 121 (2008) arXiv:0804.3466.

[16] T. Albash and C. V. Johnson, "Vortex and Droplet Engineering in Holographic Superconductors," Phys. Rev. D 80, 126009 (2009) arXiv:0906.1795.

[17] M. Montull, A. Pomarol and P. J. Silva, "The Holographic Superconductor Vortex," Phys. Rev. Lett. 103, 091601 (2009) arXiv:0906.2396 [hep-th]].

[18] K. Maeda, M. Natsuume and T. Okamura, "Vortex lattice for a holographic superconductor," Phys. Rev. D 81, 026002 (2010) arXiv:0910.4475.

[19] M. Ammon, J. Erdmenger, M. Kaminski and P. Kerner, "Superconductivity from gauge/gravity duality with flavor," Phys. Lett. B 680, 516 (2009) arXiv:0810.2316.

[20] M. Ammon, J. Erdmenger, M. Kaminski and P. Kerner, "Flavor Superconductivity from Gauge/Gravity Duality," JHEP 0910, 067 (2009) arXiv:0903.1864.

[21] D. Roychowdhury, "Effect of external magnetic field on holographic superconductors in presence of nonlinear corrections," Phys. Rev. D 86, 106009 (2012) arXiv:1211.0904 [hep-th]].

[22] D. Roychowdhury, "AdS/CFT superconductors with Power Maxwell electrodynamics: reminiscent of the Meissner effect," Phys. Lett. B 718, 1089 (2013) arXiv:1211.1612 [hep-th]]. 
[23] R. Casalbuoni and G. Nardulli, Rev. Mod. Phys. 76, 263 (2004).

[24] P. Fulde and R. A. Ferrell, "Superconductivity in a Strong Spin-Exchange Field," Phys. Rev. 135, A550 (1964).

[25] A. I. Larkin and Y. N. Ovchinnikov, "Nonuniform state of superconductors," Zh. Eksp. Teor. Fiz. 47, 1136 (1964) [Sov. Phys. JETP 20, 762 (1965)].

[26] G. T. Horowitz and J. E. Santos, "General Relativity and the Cuprates," arXiv:1302.6586 [hep-th].

[27] L. J. Romans, "Supersymmetric, cold and lukewarm black holes in cosmological Einstein-Maxwell theory," Nucl. Phys. B 383, 395 (1992) arXiv:hep-th/9203018.

[28] E. Nakano and W. -Y. Wen, "Critical magnetic field in a holographic superconductor," Phys. Rev. D 78, 046004 (2008) arXiv:0804.3180.

[29] J. Alsup, E. Papantonopoulos and G. Siopsis, "FFLO States in Holographic Superconductors," arXiv:1208.4582.

[30] J. Alsup, E. Papantonopoulos and G. Siopsis, "A Novel Mechanism to Generate FFLO States in Holographic Superconductors," Phys. Lett. B 720, 379 (2013) arXiv:1210.1541 [hep-th]].

[31] G. Siopsis and J. Therrien, "Analytic Calculation of Properties of Holographic Superconductors," JHEP 1005, 013 (2010) arXiv:1003.4275. 